\title{
Atypical desquamation in a 2.5-year-old boy with Kawasaki disease: A case report
}

Ali Adib ${ }^{1}$, Ali Fazel ${ }^{2}$, Seyed Hesamedin Nabavizadeh ${ }^{3}$, Sohaila Alyasin ${ }^{4}$, Sara Kashef ${ }^{5}$

${ }^{1}$ Student Research Committee, Shiraz University of Medical Sciences, Shiraz, Iran

${ }^{2}$ MD., Allergy Research Center, Shiraz University of Medical Sciences, Shiraz, Iran

${ }^{3}$ MD., Allergy and Clinical Immunologist, Professor, Allergy Research Center, Shiraz University of Medical Sciences, Shiraz, Iran

${ }^{4}$ MD., Allergy and Clinical Immunologist, Professor, Allergy Research Center, Shiraz University of Medical Sciences, Shiraz, Iran

${ }^{5}$ MD., Allergy and Clinical Immunologist, Professor, Department of Immunology and Allergy, Shiraz University of Medical Sciences, Shiraz, Iran

\section{Type of article: Case report}

\begin{abstract}
Kawasaki disease (KD) is a vasculitis that mostly affects children under 5 years of age. This article presents a 2.5-year-old boy who presented with 6 days of fever, generalized maculopapular rash, bilateral non-exudative conjunctivitis, cracked lips, right cervical lymphadenopathy, erythematous extremities, and perianal desquamation. Laboratory studies showed leukocytosis and sterile pyuria. Because diagnosis of KD was proved, oral acetylsalicylic acid with the anti-inflammatory dose and intravenous immunoglobulin were started for him. On the seventh day of admission time, he developed desquamation and erythema on the site of his right cervical lymphadenopathy as well as periungual scaling. About three weeks after starting the treatment, scaling of the cervical lymphadenopathy and periungual area stopped. Echocardiography was performed for him three times: at the time of diagnosis, four weeks, and 6 months later and revealed normal coronary arteries. We report this sign, desquamation on the site of cervical lymphadenopathy, as a new finding.

Keywords: Kawasaki disease, Cervical lymphadenopathy, Desquamation
\end{abstract}

\section{Introduction}

Kawasaki disease (KD) is a vasculitis of medium-size vessels. It was first described by Tomisaku Kawasaki in 1967 as infantile febrile mucocutaneous lymph node syndrome (1). The etiology of KD is relatively unknown, but an infectious insult in genetically predisposed patients has been found to play a role in this disease $(2,3)$. Innate and adaptive immune systems have been found to be involved in KD (4). It usually affects children under the age of 5 years old (5). KD is mostly a disease in Asian countries (6). There is no specific laboratory test for it, and its diagnosis is mostly based on its clinical criteria (7). KD has been responsible for acquired heart diseases in children of developed countries (8). Coronary artery aneurism is the most dangerous complication of KD (7). Diagnosis of $\mathrm{KD}$ is based on its clinical manifestations, which includes a high-grade fever that lasts five days with four of the following criteria: mucosal changes (erythema, strawberry tongue), bilateral non-exudative conjunctivitis, cervical lymphadenopathy, polymorphous systemic rash, and extremity changes (9). Extremity changes include erythema and edema of hands and feet in acute phase. This article presents a boy who had KD with a rare finding, which was desquamation on cervical lymphadenopathy in the subacute phase of the course of his disease.

\section{Corresponding author:}

Dr. Ali Fazel, Allergy Research Center, Shiraz University of Medical Sciences, Shiraz, Iran.

Tel: +98.9173440539, Email: afazel@sums.ac.ir

Received: April 21, 2016, Accepted: July 28, 2016, Published: February 2017

iThenticate screening: July 21, 2016, English editing: September 20, 2016, Quality control: December 10, 2016

(c) 2017 The Authors. This is an open access article under the terms of the Creative Commons Attribution-NonCommercialNoDerivs License, which permits use and distribution in any medium, provided the original work is properly cited, the use is non-commercial and no modifications or adaptations are made. 


\section{Case presentation}

\subsection{History}

A 2.5-year-old boy was admitted to Namazi Hospital of Shiraz in June 2014 with a history of high-grade fever from 6 days prior to admission. He had received acetaminophen, but no improvement was achieved. After 48 hours, he had developed generalized rash and conjunctivitis.

\subsection{Clinical presentations}

He was febrile on admission time $\left(37.9^{\circ} \mathrm{C}\right.$ axillary). On physical examination, generalized maculopapular rash was found. He had right cervical lymphadenopathy $(4 \mathrm{~cm} \times 2 \mathrm{~cm})$, bilateral non-exudative conjunctivitis, erythematous tympanic membrane, strawberry tongue, and dry cracked lips (Figure 1). His hands and feet were erythematous. Also, perianal desquamation was found.

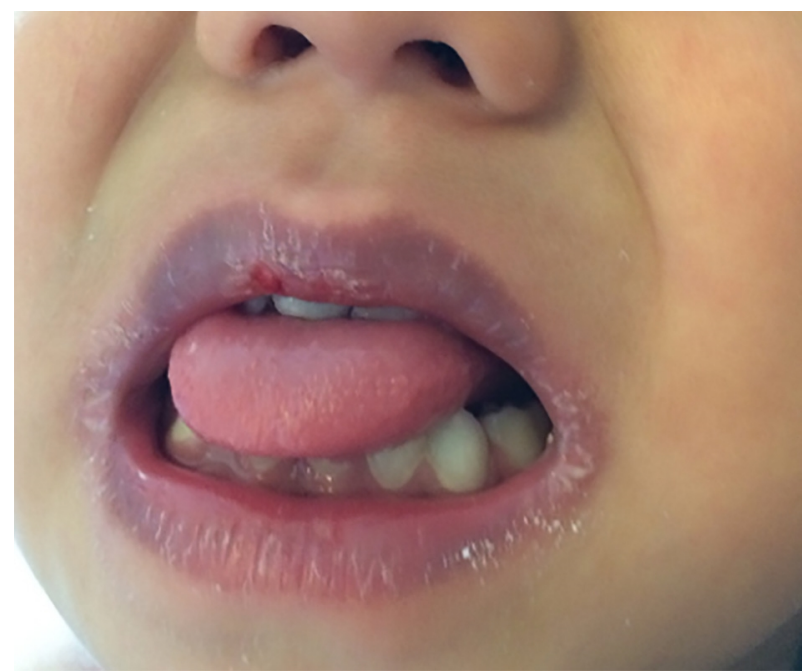

Figure 1. Cracked lips and strawberry tongue.

\subsection{Laboratory findings}

On admission time, hematological studies showed leukocytosis: white blood cell count of $19700 / \mathrm{mm}^{3}(57 \%$ neutrophils, 34\% lymphocytes, 9\% mixed), hemoglobin of $11.9 \mathrm{~g} / \mathrm{dl}$, red blood cell count of $4800000 / \mathrm{mm}^{3}$, mean corpuscular volume of $79 \mathrm{fl}$, and a platelet count of $406000 / \mathrm{mm}^{3}$. Erythrocyte sedimentation rate was $52 \mathrm{~mm} / \mathrm{h}$ and $\mathrm{C}$-reactive protein level was $34 \mathrm{mg} / \mathrm{dl}$ (negative $<6 \mathrm{mg} / \mathrm{dl}$ ). Urine analysis and urine culture were performed, and sterile pyuria was found (WBC: 25-30 and negative culture).

\subsection{Treatment}

Diagnosis of Kawasaki disease was proved by clinical criteria supporting laboratory findings. He received a slow infusion of a single dose of intravenous immunoglobulin (IVIG; $2 \mathrm{~g} / \mathrm{kg}$ ). Also, oral acetylsalicylic acid with the antiinflammatory dose $(80 \mathrm{mg} / \mathrm{kg} /$ day $)$ was started for him.

\subsection{Outcome}

Echocardiography was done, showing normal coronary arteries and normal structure and function of the heart. Electrocardiogram was normal. After seven days of admission, he developed desquamation and erythema on the site of his cervical lymphadenopathy (Figure 2). Periungual desquamation was found, too. Three weeks after starting treatment for him, scaling on the site of the right cervical lymphadenopathy was stopped, but mild erythema persisted (Figure 2). Also, desquamation of his periungual area was stopped about three weeks after treatment was started.

\subsection{Follow-up}

He was followed-up with, and after one month of treatment, the erythema of his neck disappeared. His disease was clinically improved. Laboratory investigations were as follows: white blood cell count of 12000/mm, hemoglobin of $12.7 \mathrm{~g} / \mathrm{dl}$, and platelet count of $392000 / \mathrm{mm}^{3}$. Erythrocyte sedimentation rate was $18 \mathrm{~mm} / \mathrm{h}$. Echocardiography was 
done for him again at that time and showed no coronary artery disease. About six months later, echocardiography was repeated for him, and it was normal.
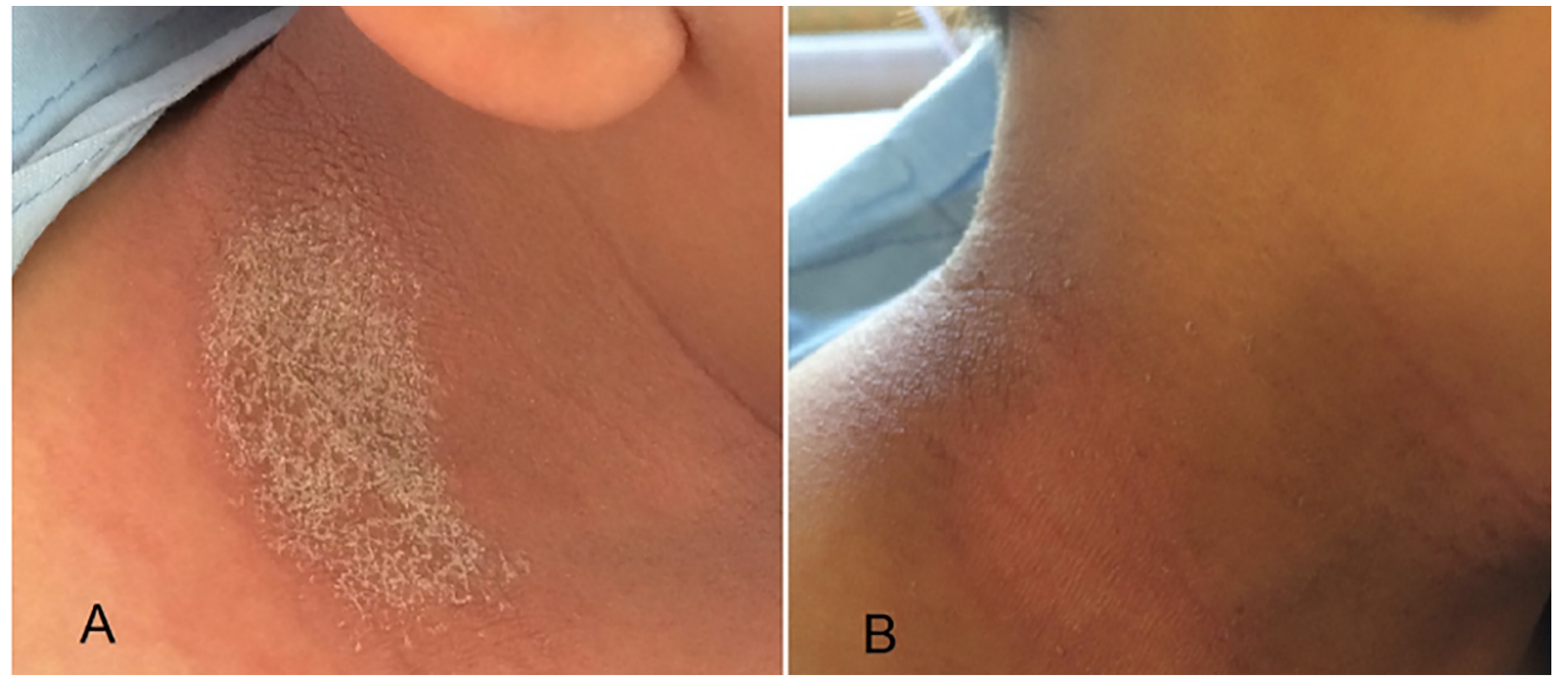

Figure 2. A. desquamation and erythema on the site of right cervical lymphadenopathy. B. disappearing of scaling and persistence of erythema.

\section{Discussion}

$\mathrm{KD}$ is an immunologic-based disease. KD may be difficult to diagnose when the patient presents with atypical manifestations. Also, when the patient does not fulfill all KD diagnostic criteria, he/she may become misdiagnosed (10). Desquamation in KD is expected on the perianal area at early stages of the disease period or on the periungual area after 2-3 weeks of the onset of fever (6). Our patient presented with scaling on the site of his right cervical lymphadenopathy as well as the mentioned routine sites. Shredding-type desquamation can be associated with disorders such as scarlet fever, leptospirosis, staphylococcal toxic shock syndrome, recurrent perineal erythema, and drug eruption (11). However, based on the clinical manifestations, laboratory findings, and the response to treatment, the only diagnosis for our patient was KD. Atypical sites of desquamation in KD have rarely been previously reported. One was a 21-year-old male Caucasian patient who presented with a diffuse maculopapular and erythema multiform-like eruption, non-exudative bilateral conjunctivitis, injected lips, strawberry tongue, and symmetrical widespread lymphadenopathy. He developed scaling of his face as well as his hands about 6 days after diagnosis (12). Another one was a 5-year-old girl presenting with the clinical manifestations of KD. She had developed periungual desquamation and fine scaling, on the skin over the dorsum of her feet (13). However, desquamation of the face or dorsum of her feet seemed to be an extension of the scaling of lips or periungual area, respectively. According to the fact that diagnosis of $\mathrm{KD}$ is mainly based on its clinical criteria, any validated novel clinical sign, which is associated with $\mathrm{KD}$, can help us with early diagnosis. Based on our PubMed search, no previous article described a case with desquamation on the site of cervical lymphadenopathy as an associated sign.

\section{Conclusions}

Our patient presented with an atypical site of desquamation. We report this sign as a new finding. It is possible that, in the future, cervical lymphadenopathy becomes accepted as a site of desquamation beside the periungual and perianal area.

\section{Acknowledgments:}

The authors would like to thank the Center for Development of Clinical Research of Namazi Hospital and Dr. Nasrin Shokrpour for editorial assistance.

\section{Conflict of Interest:}

There is no conflict of interest to be declared.

Authors' contributions:

All authors contributed to this project and article equally. All authors read and approved the final manuscript. 


\section{References:}

1) Kawasaki T, Naoe S. History of Kawasaki disease. Clin Exp Nephrol. 2014; 18(2): 301-4. doi: 10.1007/s10157-013-0877-6. PMID: 24595558.

2) Mortazavi HR, Amin R, Alyasin S, Kashef S, Karimi MH, Babaei M, et al. Down-regulation of TLR2, 3, 9 and Signaling Mediators, MyD88 and TRIF, Gene Transcript Levels in Patients with Kawasaki Disease Treated with IVIG. Iran J Allergy Asthma Immunol. 2015; 14(2): 188-97.

3) Ajami G, Aflaki K, Alyasin S, Gharesi-Fard B, Borzouee M, Amoozgar H. Distribution and Protective Role of HLAB40 in Iranian Patients with Kawasaki Disease; a Report from Southern Iran. Iran J Pediatr. 2014; 24(4): 359-64. PMID: 25755855, PMCID: PMC4339557.

4) Rasouli M, Heidari B, Kalani M. Downregulation of Th17 cells and the related cytokines with treatment in Kawasaki disease. Immunol Lett. 2014; 162(1): 269-75. doi: 10.1016/j.imlet.2014.09.017. PMID: 25277751.

5) Heidari B, Amin R, Kashef S, Alyasin S, Moghtaderi M, Aminshahidi M, et al. Expression of CD11b as an Adhesion Molecule on Neutrophils in Children with Kawasaki Disease. Iran J Allergy Asthma Immunol. 2014; 13(4): 265-70. PMID: 24659162.

6) Sánchez-Manubens J, Bou R, Anton J. Diagnosis and classification of Kawasaki disease. J Autoimmun. 2014; 48-49: 113-7. doi: 10.1016/j.jaut.2014.01.010. PMID: 24485156.

7) Greco A, De Virgilio A, Rizzo MI, Tombolini M, Gallo A, Fusconi M, et al. Kawasaki disease: An evolving paradigm. Autoimmun Rev. 2015; 14(8): 703-9. doi: 10.1016/j.autrev.2015.04.002. PMID: 25882057.

8) Kitano N, Suzuki H, Takeuchi T, Suenaga T, Kakimoto N, Shibuta S, et al. Epidemiologic features and prognostic factors of coronary artery lesions associated with Kawasaki disease based on a 13-year cohort of consecutive cases identified by complete enumeration surveys in Wakayama, Japan. J Epidemiol. 2014; 24(5): 427-34. doi: 10.2188/jea.JE20140018. PMID: 24998951, PMCID: PMC4150015.

9) Dimitriades VR, Brown AG, Gedalia A. Kawasaki disease: pathophysiology, clinical manifestations, and management. Curr Rheumatol Rep. 2014; 16(6): 423. doi: 10.1007/s11926-014-0423-x. PMID: 24744086.

10) Saguil A, Fargo M, Grogan S. Diagnosis and management of kawasaki disease. Am Fam Physician. 2015; 91(6): 365-71. PMID: 25822554.

11) Lipsker D. Clinical Examination and Differential Diagnosis of Skin Lesions. Springer. 2013; 215-7. doi: 10.1007/978-2-8178-0411-8-41.

12) Drago F, Javor S, Ciccarese G, Cozzani E, Parodi A. Complete Kawasaki Disease in a 21-Year-Old Caucasian Patient. Case Reports in Clinical Medicine. Sci Res. 2015; 4(5): 175. doi: 10.4236/crcm.2015.45035.

13) Parmar RC, Somale A, Bavdekar SB, Muranjan MN. Incomplete Kawasaki disease with recurrent skin peeling: a case report with the review of literature. J Postgrad Med. 2003; 49(1): 72-4. doi: 10.4103/00223859.924. PMID: 12865575. 